\title{
Adaptive Kernel Canonical Correlation Analysis Algorithms for Nonparametric Identification of Wiener and Hammerstein Systems
}

\author{
Steven Van Vaerenbergh, Javier Vía, and Ignacio Santamaría \\ Department of Communications Engineering, University of Cantabria, 39005 Santander, Cantabria, Spain \\ Correspondence should be addressed to Steven Van Vaerenbergh, steven@gtas.dicom.unican.es
}

Received 1 October 2007; Revised 4 January 2008; Accepted 12 February 2008

Recommended by Sergios Theodoridis

\begin{abstract}
This paper treats the identification of nonlinear systems that consist of a cascade of a linear channel and a nonlinearity, such as the well-known Wiener and Hammerstein systems. In particular, we follow a supervised identification approach that simultaneously identifies both parts of the nonlinear system. Given the correct restrictions on the identification problem, we show how kernel canonical correlation analysis (KCCA) emerges as the logical solution to this problem. We then extend the proposed identification algorithm to an adaptive version allowing to deal with time-varying systems. In order to avoid overfitting problems, we discuss and compare three possible regularization techniques for both the batch and the adaptive versions of the proposed algorithm. Simulations are included to demonstrate the effectiveness of the presented algorithm.
\end{abstract}

Copyright (C) 2008 Steven Van Vaerenbergh et al. This is an open access article distributed under the Creative Commons Attribution License, which permits unrestricted use, distribution, and reproduction in any medium, provided the original work is properly cited.

\section{INTRODUCTION}

In recent years, a growing amount of research has been done on nonlinear system identification $[1,2]$. Nonlinear dynamical system models generally have a high number of parameters although many problems can be sufficiently well approximated by simplified block-based models consisting of a linear dynamic subsystem and a static nonlinearity. The model consisting of a cascade of a linear dynamic system and a memoryless nonlinearity is known as the Wiener system, while the reversed model (a static nonlinearity followed by a linear filter) is called the Hammerstein system. These systems are illustrated in Figures 1 and 2, respectively. Wiener systems are frequently used in contexts such as digital satellite communications [3], digital magnetic recording [4], chemical processes, and biomedical engineering. Hammerstein systems are, for instance, encountered in electrical drives [5] and heat exchangers.

The past decade has seen a number of different approaches to identify these systems, which can generally be divided into three classes. First attempts followed a blackbox approach where traditionally the problem of nonlinear equalization or identification was tackled by considering nonlinear structures such as multilayer perceptrons (MLPs) [6], recurrent neural networks [3], or piecewise linear networks [7]. A second approach is the two-step method, which exploits the system structure to consecutively or alternatingly estimate the linear part and the static nonlinearity. Most proposed two-step techniques are based on predefined test signals $[8,9]$. A third method is the simultaneous estimation of both blocks, adopted, for instance, in $[10,11]$, and the iterative method in [12]. Although all above-mentioned techniques are supervised approaches (i.e., input and output signals are known during estimation), recently, there have also been a few attempts to unsupervised identification $[13,14]$.

In this paper, we focus on the problem of supervised Wiener and Hammerstein system identification, simultaneously estimating the linear and nonlinear parts. Following an idea introduced in [10], we estimate one linear filter and one memoryless nonlinearity representing the two system blocks and obtain an estimate of the signal in between these blocks. To minimize the estimation error, we use a different criterion than the one in [10]: instead of constraining the norm of the estimated filters, we fix the norm of the output signals for each block, which, as we show, leads to an algorithm that is more robust to noise. 


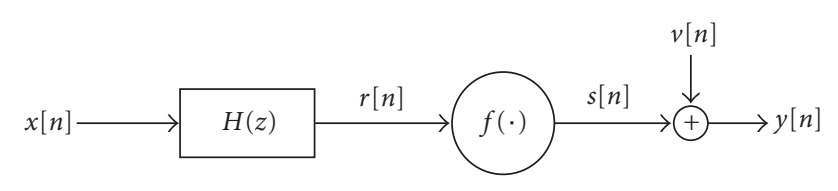

Figure 1: A Wiener system with additive noise $v[n]$.

The main contributions of this paper are twofold. First, we demonstrate how the chosen constraint leads to an implementation of the well-known kernel canonical correlation analysis (KCCA or kernel CCA) algorithm. Second, we show how the KCCA solution allows to formulate this problem as a set of two coupled least-squares (LS) regression problems that can be solved in an iterative manner, which is exploited to develop an adaptive KCCA algorithm. The resulting algorithm is capable of identifying systems that change over time. To avoid the overfitting problems that are inherent to the use of kernel methods, we discuss and compare three regularization techniques for the batch and adaptive versions of the proposed algorithm.

Throughout this paper, the following notation will be adopted: scalars will be denoted in lowercase as $x$, vectors in bold as $\mathbf{x}$ and matrices will be bold uppercase letters such as $\mathbf{X}$. Vectors will be used in column format unless otherwise mentioned, and data matrices $\mathbf{X}$ are constructed by stacking data vectors as rows of this matrix. Data points that are transformed into feature space will be represented with a tilde, for instance, $\tilde{\mathbf{x}}$. If all (row-wise stored) points of a data matrix $\mathbf{X}$ are transformed into feature space, the resulting matrix is denoted as $\tilde{\mathbf{X}}$.

The remainder of this paper is organized as follows. Section 2 describes the identification problem and the proposed identification criterion. A detailed description of the algorithm and the options to regularize its solutions are given in Section 3, which concludes with indications of how this algorithm can be used to perform full Wiener and Hammerstein system identification and equalization. The extension to the adaptive algorithm is made in Section 4 , and in Section 5 , the performance of the algorithm is illustrated by simulation examples. Finally, Section 6 summarizes the main conclusions of this work.

\section{PROBLEM STATEMENT AND PROPOSED IDENTIFICATION CRITERION}

Wiener and Hammerstein systems are two similar lowcomplexity nonlinear models. The Wiener system consists of a series connection of a linear channel and a static nonlinearity (see Figure 1). The Hammerstein system, its counterpart, is a cascade of a static nonlinearity and a linear channel (see Figure 2).

Recently, an iterative gradient identification method was presented for Wiener systems [10] that exploits the cascade structure by jointly identifying the linear filter and the inverse nonlinearity. It uses a linear estimator $\hat{H}(z)$ and a nonlinear estimator $g(\cdot)$, that, respectively, model the linear filter $H(z)$ and the inverse nonlinearity $f^{-1}(\cdot)$, as depicted in Figure 3,

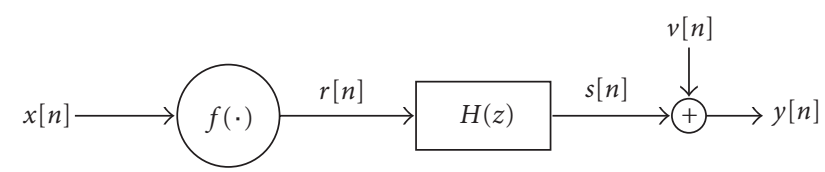

Figure 2: A Hammerstein system with additive noise.

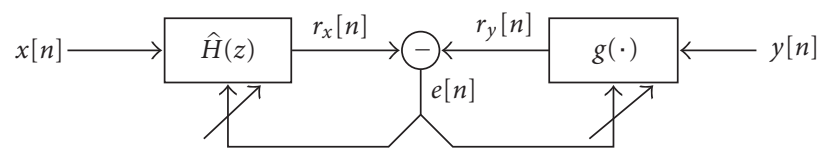

FIgURE 3: The used Wiener system identification diagram.

assuming that the nonlinearity $f(\cdot)$ is invertible in the output data range. The estimator models are adjusted by minimizing the error $e[n]$ between their outputs $r_{x}[n]$ and $r_{y}[n]$. In the noiseless case, it is possible to find estimators whose outputs correspond exactly to the reference signal $r[n]$ (up to an unknown scaling factor which is inherent to this problem).

In order to avoid the zero-solution $\hat{H}(z)=0$ and $g(\cdot)=0$, which obviously minimizes $e[n]$, a certain constraint should be applied to the solutions. For that purpose, it is instructive to look at the expanded form

$$
\|\mathbf{e}\|^{2}=\left\|\mathbf{r}_{x}-\mathbf{r}_{y}\right\|^{2}=\left\|\mathbf{r}_{x}\right\|^{2}+\left\|\mathbf{r}_{y}\right\|^{2}-2 \mathbf{r}_{x}^{T} \mathbf{r}_{y}
$$

where $\mathbf{e}, \mathbf{r}_{x}$, and $\mathbf{r}_{y}$ are vectors that contain all elements $e[n], r_{x}[n]$, and $r_{y}[n]$, respectively, with $n=1, \ldots, N$.

In [10], a linear restriction was proposed to avoid zero solutions of (1): the first coefficient of the linear filter $\hat{H}(z)$ was fixed to 1 , thus fixing the scaling factor and also the norm of all filter coefficients. With the estimated filter represented as $\mathbf{h}=\left[h_{1}, \ldots, h_{L}\right]$, the minimization problem reads

$$
\min \left\|\mathbf{r}_{x}-\mathbf{r}_{y}\right\|^{2} \quad \text { s.t. } h_{1}=1
$$

However, from (1), it is easy to see that any such restriction on the filter coefficients will not necessarily prevent the terms $\left\|\mathbf{r}_{x}\right\|^{2}$ and $\left\|\mathbf{r}_{y}\right\|^{2}$ from going to zero, hence possibly leading to noise enhancement problems. For instance, if a low-pass signal is fed into the system, the cost function (2) will not exclude the possibility that the estimated filter $\hat{H}(z)$ exactly cancels this signal, as would do a high-pass filter.

A second and more sensible restriction to minimize (1) is to fix the energy of the output signals $\mathbf{r}_{x}$ and $\mathbf{r}_{y}$ while maximizing their correlation $\mathbf{r}_{x}^{T} \mathbf{r}_{y}$, which is obtained by solving

$$
\min \left\|\mathbf{r}_{x}-\mathbf{r}_{y}\right\|^{2} \quad \text { s.t. }\left\|\mathbf{r}_{x}\right\|^{2}=\left\|\mathbf{r}_{y}\right\|^{2}=1 .
$$

Since the norms of $\mathbf{r}_{x}$ and $\mathbf{r}_{y}$ are now fixed, the zero solution is excluded per definition. To illustrate this, a direct performance comparison between batch identification algorithms based on filter coefficient constraints and this signal power constraint will be given in Section 5.1. 


\section{KERNEL CANONICAL CORRELATION ANALYSIS FOR WIENER SYSTEM IDENTIFICATION}

In this section, we will construct an identification algorithm based on the proposed signal power constraint (3). To represent the linear and nonlinear estimated filters, different approaches can be used. We will use an FIR model for the linear part of the system. For the nonlinear part, a number of parametric models can be used, including power series, Chebyshev polynomials, wavelets and piecewise linear (PWL) functions, as well as some nonparametric methods including neural networks. Nonparametric approaches do not assume that the nonlinearity corresponds to a given model, but rather let the training data decide which characteristic fits them best. We will apply a nonparametric identification approach based on kernel methods.

\subsection{Kernel methods}

Kernel methods [15] are powerful machine learning techniques built on the framework of reproducing kernel Hilbert spaces (RKHS). They are based on a nonlinear transformation $\Phi$ of the data from the input space to a high-dimensional feature space $\mathscr{H}$, where it is more likely that the problem can be solved in a linear manner [16],

$$
\Phi: \mathbb{R}^{m} \longrightarrow \mathcal{H}, \quad \Phi(\mathbf{x})=\tilde{\mathbf{x}}
$$

However, due to its high dimensionality, it is hard or even impossible to perform calculations directly in this feature space. Fortunately, scalar products in feature space can be calculated without the explicit knowledge of the nonlinear transformation $\Phi$. This is done by applying the corresponding kernel function $\kappa(\cdot, \cdot)$ on pairs of data points in the input space,

$$
\kappa\left(\mathbf{x}_{i}, \mathbf{x}_{j}\right):=\left\langle\tilde{\mathbf{x}}_{i}, \tilde{\mathbf{x}}_{j}\right\rangle=\left\langle\Phi\left(\mathbf{x}_{i}\right), \Phi\left(\mathbf{x}_{j}\right)\right\rangle
$$

This property, which is known as the "kernel trick," allows to perform any scalar product-based algorithm in the feature space by solely replacing the scalar products with the kernel function in the input space. Commonly used kernel functions include the Gaussian kernel with width $\sigma$,

$$
\kappa\left(\mathbf{x}_{i}, \mathbf{x}_{j}\right)=\exp \left(-\frac{\left\|\mathbf{x}_{i}-\mathbf{x}_{j}\right\|^{2}}{2 \sigma^{2}}\right),
$$

which implies an infinite dimensional feature space [15], and the polynomial kernel of order $p$,

$$
\kappa\left(\mathbf{x}_{i}, \mathbf{x}_{j}\right)=\left(\mathbf{x}_{i}^{T} \mathbf{x}_{j}+c\right)^{p},
$$

where $c$ is a constant.

\subsection{Identification algorithm}

To identify the linear channel of the Wiener system, we will estimate an FIR filter $\mathbf{h} \in \mathbb{R}^{L}$ whose output is given by

$$
r_{x}[n]=\mathbf{x}[n]^{T} \mathbf{h},
$$

where $\mathbf{x}[n]=[x[n], x[n-1], \ldots, x[n-L+1]]^{T} \in \mathbb{R}^{L}$ is a time-embedded vector. For the nonlinear part, we will look for a linear solution in the feature space, which corresponds to a nonlinear solution in the original space. This solution is represented as the vector $\tilde{\mathbf{h}}_{y} \in \mathbb{R}^{m^{\prime}}$, which projects the transformed data point $\tilde{\mathbf{y}}[n]=\Phi(y[n])$ onto

$$
r_{y}[n]=g(y[n])=\tilde{\mathbf{y}}[n]^{T} \tilde{\mathbf{h}}_{y}
$$

According to the representer theorem [17], the optimal $\tilde{\mathbf{h}}_{y}$ can be obtained as a linear combination of the $N$ transformed data patterns, that is,

$$
\tilde{\mathbf{h}}_{y}=\sum_{i=1}^{N} \alpha_{i} \tilde{\mathbf{y}}[i]
$$

This allows to rewrite (9) as

$$
r_{y}[n]=\sum_{i=1}^{N} \alpha_{i} \tilde{\mathbf{y}}[n]^{T} \tilde{\mathbf{y}}[i]=\sum_{i=1}^{N} \alpha_{i} \kappa(y[n], y[i])
$$

where we applied the kernel trick (5) in the second equality. Hence we obtain a nonparametric representation of the inverse nonlinearity as the kernel expansion,

$$
g(\cdot)=\sum_{i=1}^{N} \alpha_{i} \kappa(\cdot, y[i])
$$

Thanks to the kernel trick, we only need to estimate the $N$ expansion coefficients $\alpha_{i}$ instead of the $m^{\prime}$ coefficients of $\tilde{\mathbf{h}}_{y}$, for which usually holds that $N \ll m^{\prime}$.

To find these optimal linear and nonlinear estimators, it is convenient to formulate (3) in terms of matrices. By $\mathbf{X} \in \mathbb{R}^{N \times L}$, we will denote the data matrix containing $\mathbf{x}[n]$ as rows. The vector containing the corresponding outputs of the linear filter is then obtained as

$$
\mathbf{r}_{x}=\mathrm{Xh}
$$

In a similar fashion, the transformed data points $\tilde{\mathbf{y}}[n]$ can be stacked to form the transformed data matrix $\tilde{\mathbf{Y}} \in \mathbb{R}^{N \times m^{\prime}}$. The vector containing all outputs of the nonlinear estimator is

$$
\mathbf{r}_{y}=\tilde{\mathbf{Y}} \tilde{\mathbf{h}}_{y}
$$

Using (11), this can be rewritten as

$$
\mathbf{r}_{y}=\mathbf{K}_{y} \boldsymbol{\alpha},
$$

where $\mathbf{K}_{y}$ is the kernel matrix with elements $K_{y}(i, j)=$ $\kappa(y[i], y[j])$, and $\boldsymbol{\alpha}$ is a vector containing all coefficients $\alpha_{i}$. This also allows us to write $\mathbf{K}_{y}=\tilde{\mathbf{Y}} \tilde{\mathbf{Y}}^{T}$ and $\tilde{\mathbf{h}}_{y}=\tilde{\mathbf{Y}}^{T} \boldsymbol{\alpha}$.

With the obtained data representation, the minimization problem (3) is rewritten as minimizing

$$
\min \left\|\mathbf{X h}-\mathbf{K}_{y} \boldsymbol{\alpha}\right\|^{2} \quad \text { s.t. }\|\mathbf{X h}\|^{2}=\left\|\mathbf{K}_{y} \boldsymbol{\alpha}\right\|^{2}=1 .
$$


This problem is a particular case of kernel canonical correlation analysis (KCCA) [18-20] in which a linear and a nonlinear kernels are used. It has been proven [19] that minimizing (16) is equivalent to maximizing

$$
\rho=\max \frac{\mathbf{r}_{x}^{T} \mathbf{r}_{y}}{\left\|\mathbf{r}_{x}\right\|\left\|\mid \mathbf{r}_{y}\right\|}=\max _{\mathbf{h}, \boldsymbol{\alpha}} \frac{\mathbf{h}^{T} \mathbf{X}^{T} \mathbf{K}_{y} \boldsymbol{\alpha}}{\sqrt{\mathbf{h}^{T} \mathbf{X}^{T} \mathbf{X h} \boldsymbol{\alpha}^{T} \mathbf{K}_{y}^{T} \mathbf{K}_{y} \boldsymbol{\alpha}}} .
$$

If both kernels were linear, this problem would reduce to standard linear canonical correlation analysis (CCA), which is an established statistical technique to find linear relationships between two data sets [21].

The minimization problem (16) can be solved by the method of Lagrange multipliers, yielding the following generalized eigenvalue (GEV) problem [19, 22]:

$$
\frac{1}{2}\left[\begin{array}{ll}
\mathbf{X}^{T} \mathbf{X} & \mathbf{X}^{T} \mathbf{K}_{y} \\
\mathbf{K}_{y}^{T} \mathbf{X} & \mathbf{K}_{y}^{T} \mathbf{K}_{y}
\end{array}\right]\left[\begin{array}{l}
\mathbf{h} \\
\boldsymbol{\alpha}
\end{array}\right]=\beta\left[\begin{array}{cc}
\mathbf{X}^{T} \mathbf{X} & \mathbf{0} \\
\mathbf{0} & \mathbf{K}_{y}^{T} \mathbf{K}_{y}
\end{array}\right]\left[\begin{array}{l}
\mathbf{h} \\
\boldsymbol{\alpha}
\end{array}\right],
$$

where $\beta=(\rho+1) / 2$ is a parameter related to a principal component analysis (PCA) interpretation of CCA [23]. In practice, it is sufficient to solve the slightly less complex GEV

$$
\frac{1}{2}\left[\begin{array}{cc}
\mathbf{X}^{T} \mathbf{X} & \mathbf{X}^{T} \mathbf{K}_{y} \\
\mathbf{X} & \mathbf{K}_{y}
\end{array}\right]\left[\begin{array}{l}
\mathbf{h} \\
\boldsymbol{\alpha}
\end{array}\right]=\beta\left[\begin{array}{cc}
\mathbf{X}^{T} \mathbf{X} & \mathbf{0} \\
\mathbf{0} & \mathbf{K}_{y}
\end{array}\right]\left[\begin{array}{l}
\mathbf{h} \\
\boldsymbol{\alpha}
\end{array}\right] .
$$

As can be easily verified, the GEV problem (19) is transformed into (18) by premultiplication with a block-diagonal matrix containing the unit matrix and $\mathbf{K}_{y}^{T}$. Hence, any pair $(\mathbf{h}, \boldsymbol{\alpha})$ that solves (19) will also be a solution of (18).

The solution of the KCCA problem is given by the eigenvector corresponding to the largest eigenvalue of the GEV (19). However, if $\mathbf{K}_{y}$ is invertible, it is easy to see from (16) that for each $\mathbf{h}$ satisfying $\|\mathbf{X h}\|^{2}=1$, there exists an $\boldsymbol{\alpha}=$ $\mathbf{K}_{y}^{-1} \mathbf{X h}$ that solves this minimization problem and, therefore, also the GEV problem (19). This happens for sufficiently "rich" kernel functions, that is, kernels that correspond to feature spaces whose dimension $m^{\prime}$ is much higher than the number of available data points $N$. For instance, in case the Gaussian kernel is used, the feature space will have dimension $m^{\prime}=\infty$. With $N$ unknown coefficients $\alpha_{i}$, the part of (19) that corresponds to the nonlinear estimator potentially suffers from an overfitting problem. In the next section, we will discuss three different possibilities to overcome this problem by regularizing the solutions.

\subsection{Regularization techniques}

Given the different options available in literature, the solutions of (19) can be regularized by three basically different approaches. First, a small constant can be added to the diagonal of $\mathbf{K}_{y}$, corresponding to simple quadratic regularization of the problem. Second, the complexity of the matrix $\mathbf{K}_{y}$ can be limited directly by substituting it with a low-dimensional approximation. Third, a smaller subset of significant points $y[n]$ can be used to construct a sparse approximation of $\mathbf{K}_{y}$, which also yields a less complex version of this matrix. In the following, we will discuss these three regularization approaches in detail and show how they can be used to obtain three different versions of the proposed KCCA algorithm.

\subsection{1. $L_{2}$ regularization}

A common form of regularization is quadratic regularization [24], also known as ridge regression, which is often applied in kernel CCA [18-20]. It consists in restricting the $L_{2}$ norm of the solution $\widetilde{\mathbf{h}}_{y}$. The second restriction in (16) then becomes $\left\|\mathbf{K}_{y} \boldsymbol{\alpha}\right\|^{2}+c\left\|\tilde{\mathbf{h}}_{y}\right\|^{2}=1$, where $c$ is a small constant. Introducing the regularized kernel matrix $\mathbf{K}_{y}^{\mathrm{reg}}=\mathbf{K}_{y}+c \mathbf{I}$, where $\mathbf{I}$ is the identity matrix, the regularized version of (17) is obtained as

$$
\rho=\max _{\mathbf{h}, \boldsymbol{\alpha}} \frac{\mathbf{h}^{T} \mathbf{X}^{T} \mathbf{K}_{y} \boldsymbol{\alpha}}{\sqrt{\mathbf{h}^{T} \mathbf{X}^{T} \mathbf{X h}\left(\boldsymbol{\alpha}^{T} \mathbf{K}_{y}^{T} \mathbf{K}_{y}^{\mathrm{reg}} \boldsymbol{\alpha}\right)}},
$$

and the corresponding GEV problem now becomes [25]

$$
\frac{1}{2}\left[\begin{array}{cc}
\mathbf{X}^{T} \mathbf{X} & \mathbf{X}^{T} \mathbf{K}_{y} \\
\mathbf{X} & \mathbf{K}_{y}
\end{array}\right]\left[\begin{array}{l}
\mathbf{h} \\
\boldsymbol{\alpha}
\end{array}\right]=\beta\left[\begin{array}{cc}
\mathbf{X}^{T} \mathbf{X} & \mathbf{0} \\
\mathbf{0} & \mathbf{K}_{y}^{\mathrm{reg}}
\end{array}\right]\left[\begin{array}{l}
\mathbf{h} \\
\boldsymbol{\alpha}
\end{array}\right] .
$$

\subsubsection{Low-dimensional approximation}

The complexity of the kernel matrix can be reduced by performing principal component analysis (PCA) [26], which results in a kernel PCA technique [27]. This involves obtaining the first $M$ eigenvectors $\mathbf{v}_{i}$ and eigenvalues $s_{i}$ of the kernel matrix $\mathbf{K}_{y}$, for $i=1, \ldots, M$, and constructing the approximated kernel matrix

$$
\mathbf{V} \Sigma \mathbf{V}^{T} \approx \mathbf{K}_{y}
$$

where $\Sigma$ is a diagonal matrix containing the $M$ largest eigenvalues $s_{i}$, and $\mathbf{V}$ contains the corresponding eigenvectors $\mathbf{v}_{i}$ columnwise. Introducing $\overline{\boldsymbol{\alpha}}=\mathbf{V}^{T} \boldsymbol{\alpha}$ as the projection of $\boldsymbol{\alpha}$ onto the $M$-dimensional subspace spanned by the eigenvectors $\mathbf{v}_{i}$, the GEV problem (19) reduces to

$$
\frac{1}{2}\left[\begin{array}{cc}
\mathbf{X}^{T} \mathbf{X} & \mathbf{X}^{T} \mathbf{V} \Sigma \\
\mathbf{V}^{T} \mathbf{X} & \Sigma
\end{array}\right]\left[\begin{array}{l}
\mathbf{h} \\
\overline{\boldsymbol{\alpha}}
\end{array}\right]=\beta\left[\begin{array}{cc}
\mathbf{X}^{T} \mathbf{X} & \mathbf{0} \\
\mathbf{0} & \Sigma
\end{array}\right]\left[\begin{array}{l}
\mathbf{h} \\
\overline{\boldsymbol{\alpha}}
\end{array}\right],
$$

where we have exploited the fact that $\mathbf{V}^{T} \mathbf{V}=\mathbf{I}$.

\subsubsection{Sparsification of the solution}

A third approach consists in finding a subset of $M$ data points $d[i]=y\left[n_{i}\right], i=1, \ldots, M$ whose images in feature space $\tilde{\mathbf{d}}[i]$ represent the remaining transformed points $\tilde{\mathbf{y}}[n]$ sufficiently well [28]. Once a "dictionary" of points $d$ [ $i$ ] is found according to a reasonable criterion, the complete set of data points $\tilde{\mathbf{Y}}$ can be expressed in terms of the transformed dictionary as $\tilde{\mathbf{Y}} \approx \mathbf{A} \tilde{\mathbf{D}}$, where $\mathbf{A} \in \mathbb{R}^{N \times M}$ contains the coefficients of these approximate linear combinations, and $\widetilde{\mathbf{D}} \in \mathbb{R}^{M \times m^{\prime}}$ contains the points $\tilde{\mathbf{d}}[i]$ row-wise. This also reduces the expansion coefficients vector to $\hat{\boldsymbol{\alpha}}=\mathbf{A}^{T} \boldsymbol{\alpha}$, which now contains $M$ elements. Introducing the reduced kernel matrix $\widehat{\mathbf{K}}_{y}=\widetilde{\mathbf{D}} \widetilde{\mathbf{D}}^{T}$, 
the following approximation can be made:

$$
\mathbf{K}_{y}=\tilde{\mathbf{Y}} \tilde{\mathbf{Y}}^{T} \approx \mathbf{A} \hat{\mathbf{K}}_{y} \mathbf{A}^{T} .
$$

Substituting $\mathbf{K}_{y}$ for $\mathbf{A} \hat{\mathbf{K}}_{y} \mathbf{A}^{T}$ in the minimization problem (16) leads to the the GEV

$$
\frac{1}{2}\left[\begin{array}{ll}
\mathbf{X}^{T} \mathbf{X} & \mathbf{X}^{T} \mathbf{A} \hat{\mathbf{K}}_{y} \\
\mathbf{A}^{T} \mathbf{X} & \mathbf{A}^{T} \mathbf{A} \hat{\mathbf{K}}_{y}
\end{array}\right]\left[\begin{array}{l}
\mathbf{h} \\
\hat{\boldsymbol{\alpha}}
\end{array}\right]=\beta\left[\begin{array}{cc}
\mathbf{X}^{T} \mathbf{X} & \mathbf{0} \\
\mathbf{0} & \mathbf{A}^{T} \mathbf{A} \hat{\mathbf{K}}_{y}
\end{array}\right]\left[\begin{array}{l}
\mathbf{h} \\
\widehat{\boldsymbol{\alpha}}
\end{array}\right] .
$$

In [28], a sparsification procedure was introduced to obtain such a dictionary of significant points, albeit in an online manner in the context of kernel recursive least-squares regression (KRLS or kernel RLS). It was also shown that this online sparsification procedure is related to kernel PCA. In Section 4, we will adopt this online procedure to regularize the adaptive version of the proposed KCCA algorithm.

\subsection{A Unified approach to Wiener and Hammerstein system identification and equalization}

To identify the linear channel and the inverse nonlinearity of the Wiener system, any of the regularized GEV problems (21), (23), or (25) can be solved. Moreover, given the symmetric structure of the Wiener and Hammerstein systems (see Figures 1 and 2), it should be clear that the same approach can be applied to identify the blocks of the Hammerstein system. To do so, the linear and nonlinear estimators of the proposed kernel CCA algorithm need to be switched. The resulting Hammerstein system identification algorithm estimates the direct static nonlinearity and the inverse linear channel, which is retrieved as an FIR filter.

Full identification of an unknown system provides an estimate of the system output given a certain input signal. To fully identify the Wiener system, the presented KCCA algorithm needs to be complemented with an estimate of the direct nonlinearity $f(\cdot)$. This nonlinearity can be obtained by applying any nonlinear regression algorithm on the signal in between the two blocks (whose estimate is provided by the KCCA-based algorithm) and the given output signal y. In particular, to stay within the scope of this paper, we propose to obtain $\hat{f}(\cdot)$ as another kernel expansion as follows:

$$
\hat{f}(\cdot)=\sum_{i=1}^{N} \beta_{i} \kappa\left(\cdot, r_{x}[i]\right) .
$$

Note that in practice, this nonlinear regression should use $\mathbf{r}_{x}$ as input signal since this will be less influenced by the additive noise $\mathbf{v}$ on the output than $\mathbf{r}_{y}$, the other estimate of the reference signal. In Section 5, the full identification process is illustrated with some examples.

Apart from Wiener system identification, a number of algorithms can be based directly on the presented KCCA algorithm. In case of the Hammerstein system, KCCA already obtains an estimate of the direct nonlinearity and the inverse linear channel. To fully identify the Hammerstein system, the direct linear channel needs to be estimated, which can be done by applying standard filter inversion techniques [29].
At this point, it is interesting to note that the inversion of the estimated linear filter can also be used in equalization of the Wiener system [22], where the KCCA algorithm already obtained the inverse of the nonlinear block. To come full circle, a Hammerstein system equalization algorithm can be constructed based on the inverse linear channel estimated by KCCA and the inverse nonlinearity that can be obtained by performing nonlinear regression on the appropriate signals. A detailed study of these derived algorithms will be a topic for future research.

\section{ADAPTIVE SOLUTION}

In a number of situations, it is desirable to have an adaptive algorithm that can update its solution according to newly arriving data. Standard scenarios include problems where the amount of data is too high to apply a batch algorithm. An adaptive (or online) algorithm can calculate the solution to the entire problem by improving its solution on a sample-bysample basis, thereby maintaining a low computational complexity. Another scenario happens when the observed problem or system is time varying. Instead of improving its solution, the online algorithm must now adjust its solution to the changing conditions. In this second case, the algorithm must be capable of excluding the influence of less recent data, which can be done, for instance, by introducing a forgetting factor.

In this section, we discuss an adaptive version of kernel CCA which can be used for online identification of Wiener and Hammerstein systems.

\subsection{Formulation of KCCA as coupled RLS problems}

The special structure of the GEV problem (19) has recently been exploited to obtain efficient CCA and KCCA algorithms $[22,30,31]$. Specifically, this GEV problem can be viewed as two coupled least-squares regression problems

$$
\begin{gathered}
\beta \mathbf{h}=\left(\mathbf{X}^{T} \mathbf{X}\right)^{-1} \mathbf{X}^{T} \widehat{\mathbf{r}}, \\
\beta \mathbf{K}_{y} \boldsymbol{\alpha}=\hat{\mathbf{r}},
\end{gathered}
$$

where $\widehat{\mathbf{r}}=\left(\mathbf{r}_{x}+\mathbf{r}_{y}\right) / 2=\left(\mathbf{X h}+\mathbf{K}_{y} \boldsymbol{\alpha}\right) / 2$. This idea has been used in $[22,32]$ to develop an algorithm based on the solution of these regression problems iteratively: at each iteration $t$, two LS regression problems are solved using

$$
\widehat{\mathbf{r}}(t)=\frac{\mathbf{r}_{x}(t)+\mathbf{r}_{y}(t)}{2}=\frac{\mathbf{X h}(t-1)+\mathbf{K}_{y} \boldsymbol{\alpha}(t-1)}{2}
$$

as desired output.

Furthermore, this LS regression framework was exploited directly to develop an adaptive CCA algorithm based on the recursive least-squares algorithm (RLS), which was shown to converge to the CCA solution [32]. For Wiener and Hammerstein system identification, the adaptive solution of (27) can be obtained by coupling one linear RLS algorithm with one kernel RLS algorithm. Before describing the complete adaptive algorithm in detail, we first review the different options that exist to implement kernel RLS. 


\subsection{Kernel recursive least-squares regression}

As is the case with all online kernel algorithms, the design of a kernel RLS algorithm presents some crucial difficulties [33] that are not present in standard online settings for linear methods. Apart from the previously mentioned problems that arise from overfitting, an important bottleneck is the complexity of the functional representation of kernelbased estimators. The representer theorem [17] implies that the number of kernel functions grows linearly with the number of observations. For a kernel RLS algorithm, this translates into an algorithm based on a growing kernel matrix, implying a growing computational and memory complexity. To limit the number of observations used at each time step and to prevent overfitting at the same time, the three previously discussed forms of regularization can be redefined in an online context. For each resulting type of kernel RLS, the update of the solution is discussed and a formula to obtain a new output estimate is given, both of which are necessary for online operation.

\subsubsection{Sliding-window kernel $R L S$ with $L_{2}$ regularization}

In $[25,34]$, a kernel RLS algorithm was presented that performed online kernel RLS regression applying standard regularization of the kernel matrix. Compared to standard linear RLS, which can be extended to include both regularization and a forgetting factor, in kernel RLS, it is more difficult to simultaneously apply $L_{2}$ regularization and lower the influence of older data points. Therefore, this algorithm uses a sliding window to straightforwardly fix the number of observations to take into account. This approach is able to track changes of the observed system, and it is easy to implement. However, its computational complexity is $O\left(N_{w}^{2}\right)$, where $N_{w}$ is the number of data points in the sliding window, and hence it presents a tradeoff between performance and computational cost.

The sliding window used in this method consists of a buffer that retains the last $N_{w}$ input data points on one hand, represented by $\mathbf{y}=\left[y[n], \ldots, y\left[n-N_{w}+1\right]\right]^{T}$, and the last $N_{w}$ desired output data samples $\widehat{\mathbf{r}}=\left[\hat{r}[n], \ldots, \hat{r}\left[n-N_{w}+1\right]\right]^{T}$ on the other hand. The transformed data $\tilde{\mathbf{Y}}$ is used to calculate the regularized kernel matrix $\mathbf{K}_{y}^{\text {reg }}=\tilde{\mathbf{Y}} \tilde{Y}^{T}+c \mathbf{I}$, which leads to the following solution to the LS regression problem:

$$
\boldsymbol{\alpha}=\left(\mathbf{K}_{y}^{\mathrm{reg}}\right)^{-1} \widehat{\mathbf{r}} \text {. }
$$

In an online setup, a new input-output pair $\{y[n], \hat{r}[n]\}$ is received at each time step. The sliding-window approach consists in adding this new data point to the buffers $\mathbf{y}$ and $\hat{\mathbf{r}}$, and discarding the oldest data point. A method to efficiently update the inverse regularized kernel matrix is discussed in [25]. Then, given an estimate of $\boldsymbol{\alpha}$, the estimated output $r_{y}$ corresponding to a new input point $y$ can be calculated as

$$
r_{y}=\sum_{i=1}^{N_{w}} \alpha_{i} \tilde{\mathbf{y}}_{i} \tilde{\mathbf{y}}=\sum_{i=1}^{N_{w}} \alpha_{i} \kappa\left(y_{i}, y\right)=\mathbf{k}_{\mathbf{y}}^{T} \boldsymbol{\alpha},
$$

where $\mathbf{k}_{\mathbf{y}}$ is a vector containing the elements $\kappa\left(y_{i}, y\right)$, and $y_{i}$ corresponds to the points in the input data buffer. This allows to obtain the identification error of the algorithm.
When this algorithm is used as the kernel RLS algorithm in the adaptive kernel CCA framework for Wiener system identification, the coupled LS regression problems (27) become

$$
\begin{gathered}
\beta \mathbf{h}=\left(\mathbf{X}^{T} \mathbf{X}\right)^{-1} \mathbf{X}^{T} \widehat{\mathbf{r}}, \\
\beta \boldsymbol{\alpha}=\left(\mathbf{K}_{y}^{\mathrm{reg}}\right)^{-1} \widehat{\mathbf{r}} .
\end{gathered}
$$

\subsubsection{Online kernel PCA-based RLS}

A second possible implementation of kernel RLS is obtained by using a low-dimensional approximation of the kernel matrix, for which we will adopt the notations from Section 3.3.2. Recently, an online implementation of the kernel PCA algorithm was proposed [35], that updates the eigenvectors $\mathbf{V}$ and eigenvalues $s_{i}$ of the kernel matrix $\mathbf{K}_{y}$ as new data points are added. It has the possibility to exclude the influence of older observations in a sliding-window fashion (with window length $N_{w}$ ), which makes it suitable for time-varying problem settings. Its computational complexity is $O\left(N_{w} M^{2}\right)$.

In the adaptive kernel CCA framework for Wiener system identification, the online kernel PCA algorithm can be used to approximate the second LS regression problem from (27), leading to the following set of coupled problems:

$$
\begin{gathered}
\beta \mathbf{h}=\left(\mathbf{X}^{T} \mathbf{X}\right)^{-1} \mathbf{X}^{T} \widehat{\mathbf{r}}, \\
\beta \overline{\boldsymbol{\alpha}}=\Sigma^{-1} \mathbf{V}^{T} \hat{\mathbf{r}} .
\end{gathered}
$$

Furthermore, the estimated output $r_{y}$ of the nonlinear filter corresponding to a new input point $y$ is calculated by this algorithm as

$$
r_{y}=\sum_{i=1}^{N} \sum_{j=1}^{M} \kappa\left(y_{i}, y\right) V_{i j} \bar{\alpha}_{i}=\mathbf{k}_{\mathbf{y}}^{T} \mathbf{V} \overline{\boldsymbol{\alpha}},
$$

where $V_{i j}$ denotes the $i$ th element of the eigenvector $\mathbf{v}_{j}$.

\subsubsection{Kernel RLS with sequential sparsification}

The kernel RLS algorithm from [28] limits the kernel matrix size by means of an online sparsification procedure, which maps the data points to a reduced-size dictionary. At the same time, this approach avoids overfitting, as was pointed out in Section 3.3.3. It is computationally efficient (with $O\left(M^{2}\right), M$ being the dictionary size), but due to its lack of any kind of "forgetting mechanism," it is not truly adaptive and hence is less efficient to adapt to time-varying environments. A related iterative kernel LS algorithm was recently presented in [36].

The dictionary-based kernel RLS algorithm recursively obtains its solution by efficiently solving

$$
\widehat{\boldsymbol{\alpha}}=\left(\mathbf{A} \hat{\mathbf{K}}_{y}\right)^{\dagger} \mathbf{r}_{y}=\hat{\mathbf{K}}_{y}^{-1}\left(\mathbf{A}^{T} \mathbf{A}\right) \mathbf{A}^{T} \mathbf{r}_{y}
$$

where $\mathbf{r}_{y}$ contains all input data. After plugging this kernel RLS algorithm into (27), the coupled LS regression problems 
Initialize the RLS and KRLS algorithm.

for $n=1,2, \ldots$

Obtain the new system input-output pair $\{\mathbf{x}[n], y[n]\}$.

Compute $r_{x}[n]$ and $r_{y}[n]$, the outputs of the RLS and KRLS algorithms, respectively.

Calculate the estimated reference signal $\hat{r}[n]=\left(r_{x}[n]+r_{y}[n]\right) / 2$.

Use the input-output pairs $\{\mathbf{x}[n], \hat{r}[n]\}$ and $\{y[n], \hat{r}[n]\}$ to update the RLS and KRLS solutions $\mathbf{h}$ and $\boldsymbol{\alpha}$.

Normalize the solutions with $\beta=\|\mathbf{h}\|$, that is, $\mathbf{h} \leftarrow \mathbf{h} / \beta$ and $\boldsymbol{\alpha} \leftarrow \boldsymbol{\alpha} / \beta$.

Algorithm 1: The adaptive kernel CCA algorithm for Wiener system identification.

become

$$
\begin{gathered}
\beta \mathbf{h}=\left(\mathbf{X}^{T} \mathbf{X}\right)^{-1} \mathbf{X}^{T} \widehat{\mathbf{r}}, \\
\beta \widehat{\boldsymbol{\alpha}}=\widehat{\mathbf{K}}_{y}^{-1}\left(\mathbf{A}^{T} \mathbf{A}\right) \mathbf{A}^{T} \widehat{\mathbf{r}} .
\end{gathered}
$$

Given an estimate of $\hat{\boldsymbol{\alpha}}$, the estimated output $r_{y}$ corresponding to a new input point $y$ can be calculated as

$$
r_{y}=\sum_{i=1}^{M} \widetilde{\alpha}_{i} \kappa(d[i], y)=\mathbf{k}_{\mathbf{d y}}^{T} \hat{\boldsymbol{\alpha}},
$$

where $\mathbf{k}_{\mathbf{d y}}$ contains the kernel functions of the points in the dictionary and the data point $y$.

\subsection{Adaptive identification algorithm}

The adaptive algorithm couples a linear and a nonlinear RLS algorithms, as in (27). For the nonlinear RLS algorithm, any of the three discussed regularized kernel RLS methods can be used. The complete algorithm is summarized in Algorithm 1. Notice the normalization step at the end of each iteration, which fixes the scaling factor of the solution.

\section{EXPERIMENTS}

In this section, we experimentally test the proposed kernel CCA-based algorithms. We begin by comparing three algorithms based on different error minimization constraints, in a batch experiment. Next, we conduct a series of online identification tests including a static Wiener system, a timevarying Wiener system, and a static Hammerstein system.

To compare the performance of the used algorithms, two different MSE values can be analyzed. First, the kernel CCA algorithms' success can be measured directly by comparing the estimated signal $\hat{r}$ to the real internal signal $r$ of the system, resulting in the error $e_{r}=r-\hat{r}$. Second, as shown in Section 3.4, the proposed KCCA algorithms can be extended to perform full system identification and equalization. In that case, the identification error is obtained as the difference between estimated system output and real system output, $e_{y}=y-\hat{y}$.

The input signal for all experiments consisted of a Gaussian with distribution $\mathcal{N}(0,1)$ and to the output of the Wiener or Hammerstein system additive zero-mean white Gaussian noise was added. Two different linear channels and

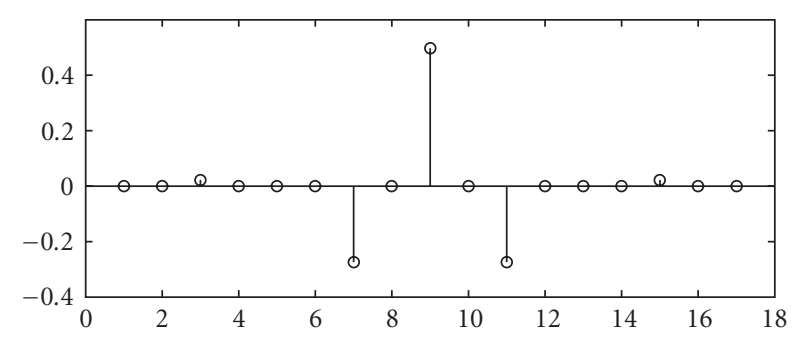

FIgURE 4: The 17 taps bandpass filter used as the linear channel in the Wiener system, generated in Matlab as fir $1(16,[0.25,0.75])$.

two different nonlinearities were used. The exact setup is specified in each experiment, and the length of the linear filter is supposed to be known in all cases. In [22], it was shown that the performance of the kernel CCA algorithm for Wiener identification is hardly affected by overestimation of the linear channel length. Therefore, if the exact filter length was not known, it could be overestimated without significant performance loss.

\subsection{Batch identification}

In the first experiment, we compare the performance of the different constraints to minimize the error $\left\|\mathbf{r}_{x}-\mathbf{r}_{y}\right\|^{2}$ between the linear and nonlinear estimates in the simultaneous identification scheme from Section 3. The identification of a static Wiener system is treated here as a batch problem, that is, all data points are available beforehand.

The Wiener system used for this setup consists of the static linear channel from [10] representing an FIR bandpass filter of 17 taps (see Figure 4) and a static nonlinearity given by $f(x)=0.2 x+\tanh (x) .500$ samples are used to identify this system.

To represent the inverse nonlinearity, a kernel expansion is used, based on a Gaussian kernel with kernel size $\sigma=0.2$. In order to avoid overfitting of the kernel matrix, $L_{2}$ regularization is applied by adding a constant $c=10^{-4}$ to its diagonal.

Three different identification approaches are applied, using different constraints to minimize the error $\|\mathbf{e}\|^{2}$. As discussed in Section 2, these constraints can be based on the filter coefficients or the signal energy. In a first approach, we apply the filter coefficient norm constraint (2) (from [10]), which fixes $h_{1}=1$. The corresponding optimal estimators 


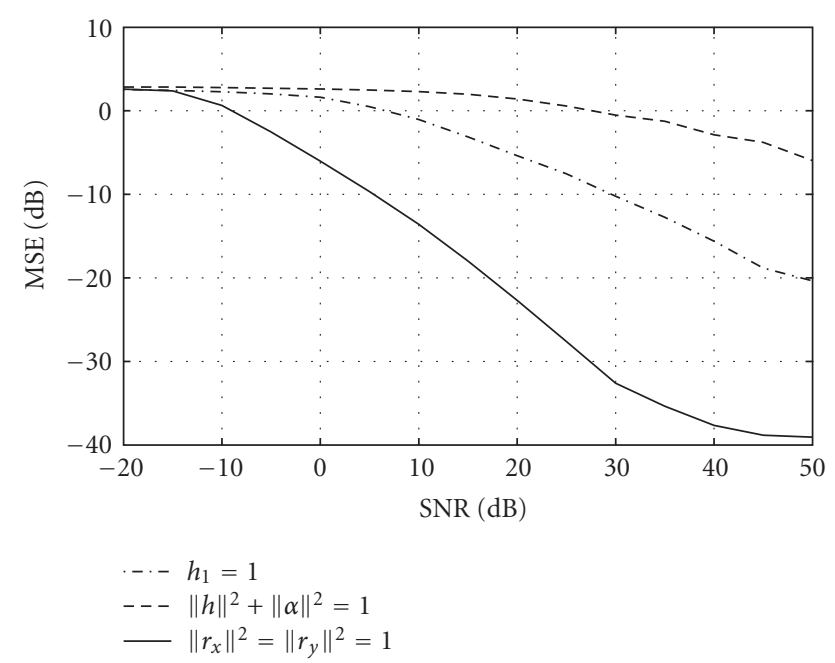

FIgURE 5: MSE $\left\|\mathbf{e}_{r}\right\|^{2}$ on the Wiener system's internal signal. The algorithms based on filter coefficient constraints (dotted and dashed lines) perform worse than the proposed KCCA algorithm (solid line), which is based on a signal power constraint.

are found by solving a simple LS problem. If, instead, we fix the filter norm $\|\mathbf{h}\|^{2}+\|\boldsymbol{\alpha}\|^{2}=1$, we obtain the following problem:

$$
\min \left\|\mathbf{r}_{x}-\mathbf{r}_{y}\right\|^{2} \quad \text { s.t. }\|\mathbf{h}\|^{2}+\|\boldsymbol{\alpha}\|^{2}=1,
$$

which, after introducing the substitutions $\mathbf{L}=\left[\mathbf{X},-\mathbf{K}_{y}\right]$ and $\mathbf{v}=\left[\mathbf{h}^{T}, \boldsymbol{\alpha}^{T}\right]^{T}$, becomes

$$
\min \|\mathbf{L v}\|_{F}=\min \left\|\mathbf{v}^{T} \mathbf{L}^{T} \mathbf{L v}\right\| \quad \text { s.t. }\|\mathbf{v}\|^{2}=1 .
$$

The solution $\mathbf{v}$ of this second approach is found as the eigenvector corresponding to the smallest eigenvalue of the matrix $\mathbf{L}^{T} \mathbf{L}$. As a third approach, we apply the signal energy-based constraint (3), which fixes $\left\|\mathbf{r}_{x}\right\|^{2}=\left\|\mathbf{r}_{y}\right\|^{2}=1$. The corresponding solution is obtained by solving the GEV (21).

In Figure 5, the performance results are shown for the three approaches and for different noise levels. To calculate the error $\mathbf{e}_{r}=\mathbf{r}-\hat{\boldsymbol{r}}$, both $\mathbf{r}$ and $\hat{\mathbf{r}}$ have been normalized to compensate for the scaling indeterminacy of the Wiener system. The MSE is obtained by averaging out $\left\|\mathbf{e}_{r}\right\|^{2}$ over 250 runs of the algorithms. As can be observed, the algorithms based on the filter coefficient constraints perform clearly worse than the proposed KCCA algorithm, which is more robust to noise.

Figure 6 compares the real inverse nonlinearity to the estimate of this nonlinearity for the solution based on the $h_{1}$ filter coefficient constraint and to the estimate obtained by regularized KCCA. For $20 \mathrm{~dB}$ of output noise, the results of the first algorithm are dominated by noise enhancement problems (Figure 6(d)). This further illustrates the advantage of the signal power constraint over the filter coefficient constraint.

In the second experiment, we compare the full Wiener system identification results for the KCCA approach to two black box neural network methods, specifically a radial basis function (RBF) network and a multilayer perceptron (MLP). The Wiener system setup and used input signal are the same as in the previous experiment.

For a fair comparison, the used solution methods should have similar complexity. Since complexity comparison is difficult due to the significant architectural differences between kernel and classic neural network approaches [15], we compare the identification methods when simply given a similar number of parameters. The KCCA algorithm requires $17 \mathrm{pa}-$ rameters to identify the linear channel and 500 parameters in its kernel expansion, totalling 517. When the RBF network and the MLP have 27 neurons in their hidden layer, they obtain a comparable total of 514 parameters, considering they use a time-delay input of length 17. For the MLP, however, better results were obtained by lowering its number of neurons, and therefore, we only assigned it 15 neurons. The RBF network was trained with a sum-squared error goal of $10^{-6}$ and the Gaussian function of its centers had a spread of 10 . The MLP used a hyperbolic tangent transfer function, and it was trained over 50 epochs with the Levenberg-Marquardt algorithm.

The results of the batch identification experiment can be seen in Figure 7. The KCCA algorithm performs best due to its knowledge of the internal structure of the system. Note that by choosing the hyperbolic tangent function as the transfer function, the MLP's structure closely resembles the used Wiener system and, therefore, also obtains good performance.

\subsection{Online identification}

In a second set of simulations, we compare the identification performance of the three adaptive kernel CCA-based identification algorithms from Section 4. In all online experiments, the optimal parameters as well as the kernel for each of the algorithms were determined by an exhaustive search.

\subsubsection{Static Wiener system identification}

The Wiener system used in this experiment contained the same linear channel as in the previous batch example, followed by the nonlinearity $f(x)=\tanh (x)$. No output noise was added in this first setup.

We applied the three proposed adaptive kernel CCAbased algorithms with the following parameters:

(i) kernel CCA with standard regularization, $c=10^{-3}$, and a sliding window of 150 samples, using the Gaussian kernel function with kernel width $\sigma=0.2$;

(ii) kernel CCA based on kernel PCA using 15 eigenvectors calculated from a 150-sample sliding window, and applying the polynomial kernel function of order 3;

(iii) kernel CCA with the dictionary-based sparsification method from [28], with a polynomial kernel function of order 3 and accuracy parameter $v=10^{-4}$. This parameter controls the level of sparsity of the solution.

The RLS algorithm used in all three cases was a standard exponentially weighted RLS algorithm [29] with a forgetting factor of 0.99 . 


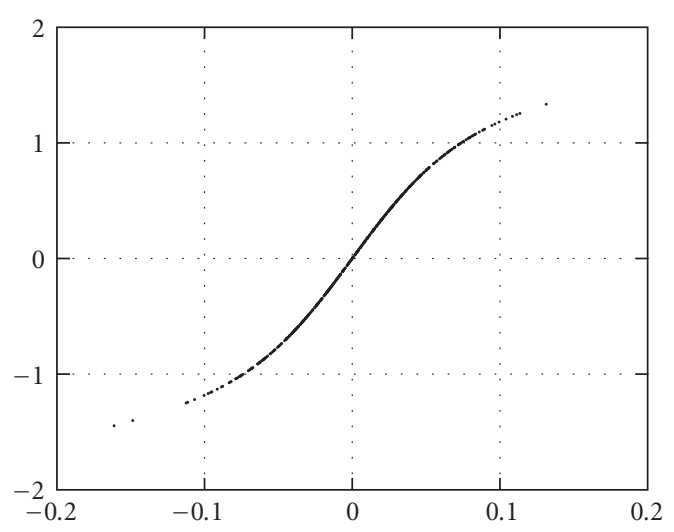

(a) $r[n]$ versus $y[n]$, no noise

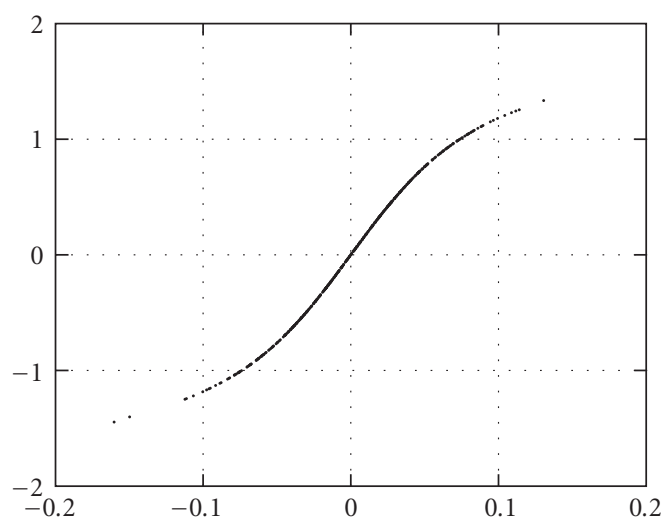

(c) Estimate with $h_{1}$ constraint, no noise

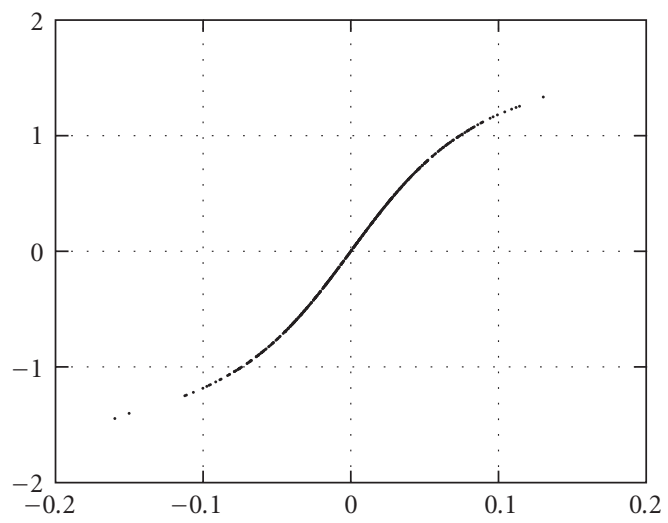

(e) KCCA estimate, no noise

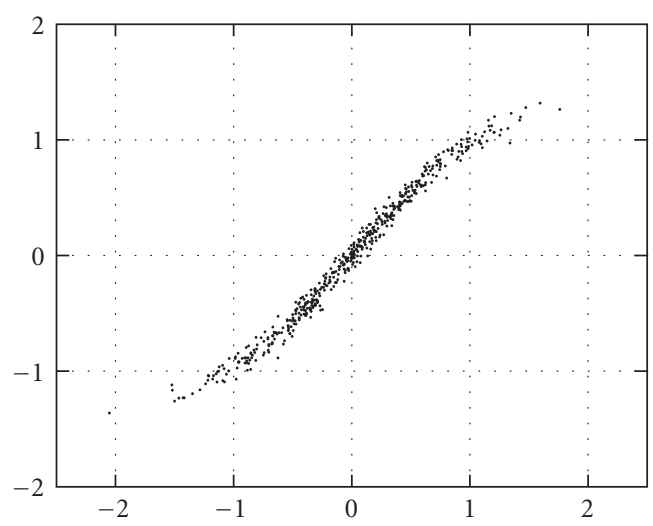

(b) $r[n]$ versus $r[n], 20 \mathrm{~dB}$ SNR

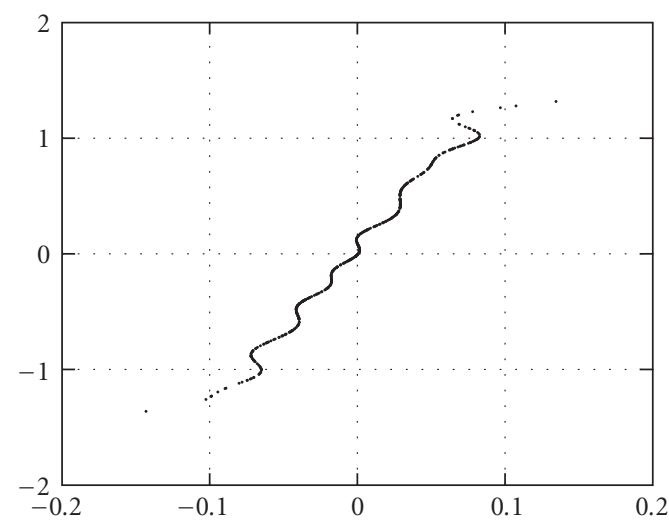

(d) Estimate with $h_{1}$ constraint, $20 \mathrm{~dB}$ SNR

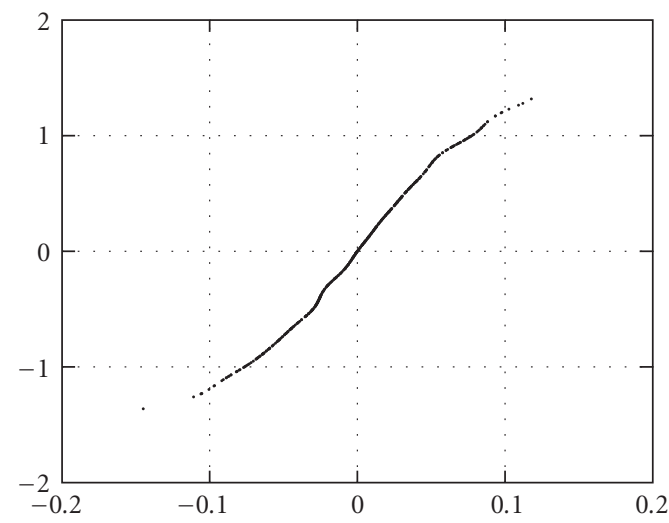

(f) KCCA estimate, $20 \mathrm{~dB}$ SNR

FIGURE 6: Estimates of the nonlinearity in the static Wiener system. The top row shows the true signal $r[n]$ versus the points $y[n]$ representing the system nonlinearity, for a noiseless case in (a) and a system that has $20 \mathrm{~dB}$ white Gaussian noise at its output in (b). The second and third row show $r_{y}[n]$ versus $y[n]$ obtained by applying the filter coefficient constraint $h_{1}=1$ and the signal power constraint (KCCA solution), respectively.

The obtained MSE $e_{r}^{2}[n]$ for the three algorithms can be seen in Figure 8. Most notable is the slow convergence of the dictionary-based kernel CCA implementation. This is explained by the fact that the used dictionary-based kernel RLS algorithm from [28] is lacking a forgetting mechanism and, therefore, it takes a large number of iterations for the influence of the initially erroneous reference signal $\hat{r}$ to decrease. The kernel PCA-based algorithm obtains its optimal perfor- mance for a polynomial kernel, while the $L_{2}$ regularized kernel CCA algorithm performs slightly better, with the Gaussian kernel.

A comparison of the results of the sliding window KCCA algorithm for different noise levels is given in Figure 9. A different Wiener system was used, with linear channel $H(z)=$ $1+0.3668 z^{-1}-0.5764 z^{-2}+0.2070 z^{-3}$ and nonlinearity $f(x)=\tanh (x)$. 


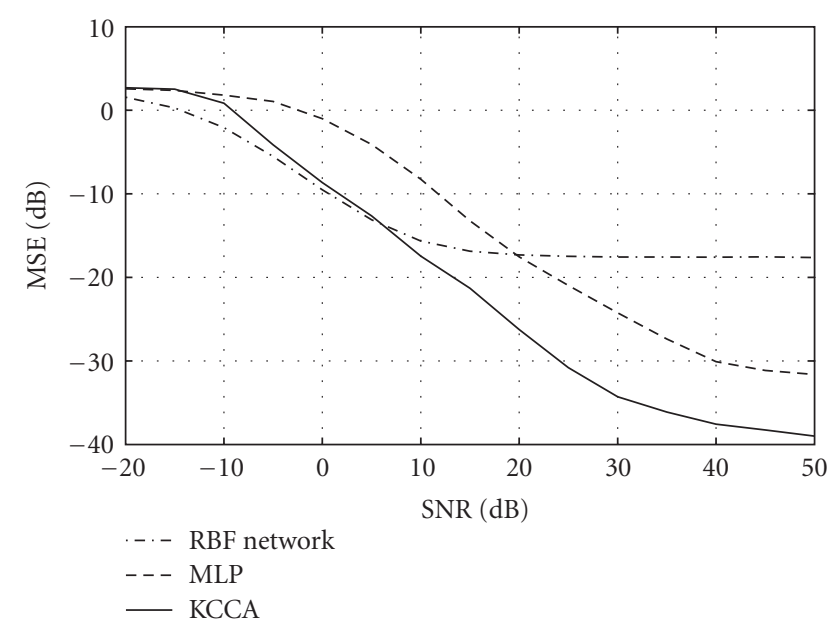

FIgURE 7: Full identification MSE $\left\|\mathbf{e}_{y}\right\|^{2}$ of the Wiener system, using two black box methods (RBF network and MLP) and the proposed KCCA algorithm.

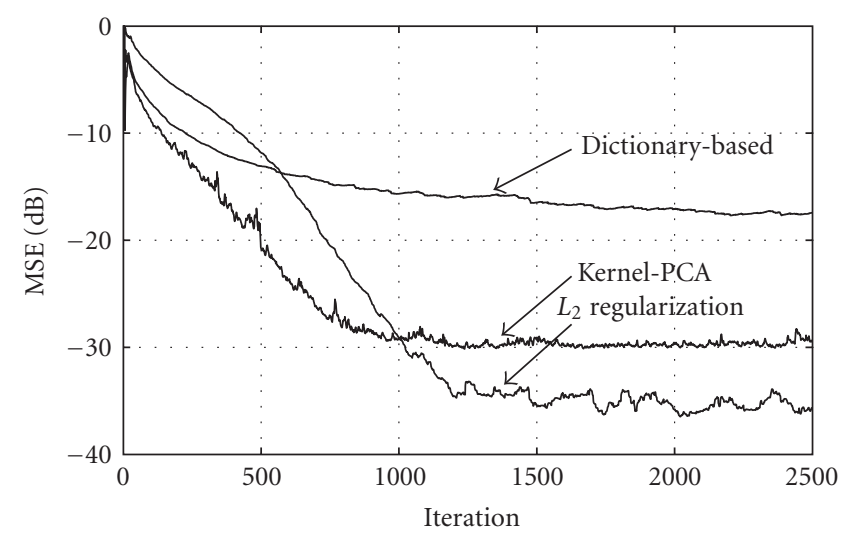

FIgURE 8: MSE $e_{r}^{2}[n]$ on the Wiener system's internal signal $r[n]$ for adaptive kernel CCA-based identification of a static noiseless Wiener system.

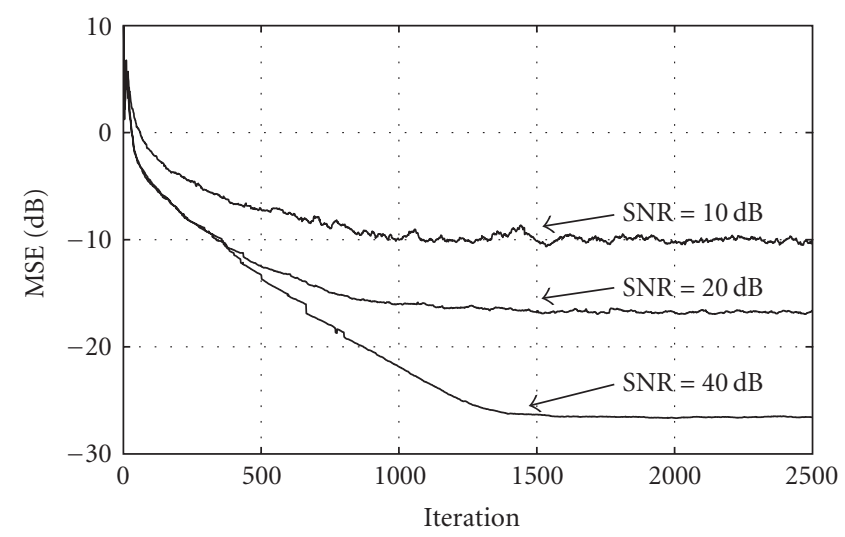

FIgURE 9: MSE $e_{r}^{2}[n]$ on the Wiener system's internal signal $r[n]$ for various noise levels, obtained by the adaptive KCCA algorithm.

Figure 10 shows the full system identification results obtained by an MLP and the proposed KCCA algorithm on this wiener system. The used MLP has learning rate 0.01 and was

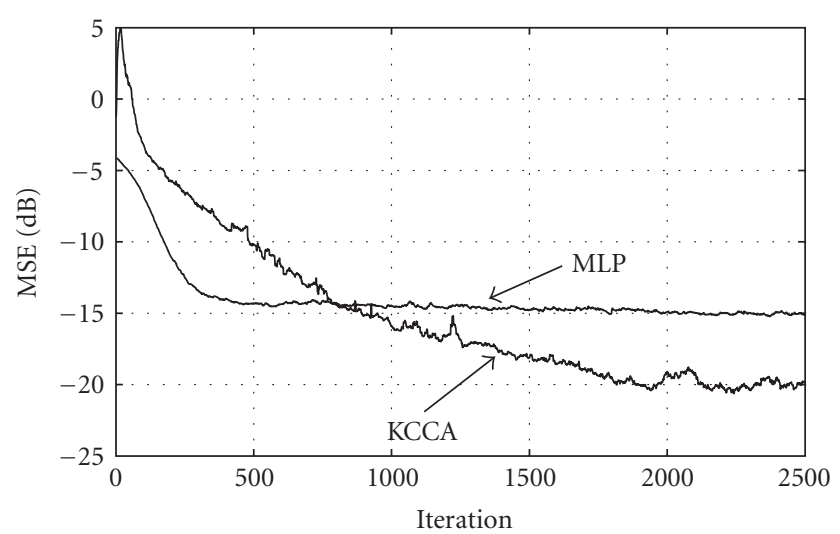

Figure 10: MSE $e_{y}^{2}[n]$ for full system identification of the Wiener system, using a black-box method (MLP) and the proposed KCCA algorithm.

trained at each iteration step with the new data point. The KCCA algorithm again has $L_{2}$ regularization with $c=10^{-3}$, $\sigma=0.2$, and a sliding window of 150 samples. Both the inverse nonlinearity and the direct nonlinearity were estimated with the sliding-window kernel RLS technique. Although this algorithm converges slower, it is clear that its knowledge of the internal structure of the Wiener system implies a considerable advantage over the black-box approach.

\subsubsection{Dynamic Wiener system identification}

In a second experiment, the tracking capabilities of the discussed algorithms were tested. Therefore, an abrupt change in the Wiener system was triggered (note that although only the linear filter is changed, the proposed adaptive identification method allows both parts of the Wiener system to be varying in time): during the first part, the Wiener system uses the 17-coefficient channel from the previous tests, but after receiving the 1000th data point, its channel is changed to $H(z)=1+0.3668 z^{-1}-0.5764 z^{-2}+0.2070 z^{-3}$. The nonlinearity was $f(x)=\tanh (x)$ in both cases. Moreover, $20 \mathrm{~dB}$ of zero-mean white Gaussian noise was added to the output of the system during the entire experiment.

The parameters of the applied identification algorithms were chosen as follows.

(i) For Kernel CCA with standard regularization, we used $c=10^{-3}$, a sliding window of 150 samples, and the polynomial kernel function of order 3 .

(ii) The Kernel CCA algorithm based on kernel PCA was used with 15 eigenvectors, a sliding window of 150 samples, and the polynomial kernel function of order 3 .

(iii) Finally, for Kernel CCA with the dictionary-based sparsification method, we used accuracy parameter $v=10^{-3}$ and a polynomial kernel function of order 3 .

The length of the estimated linear channel was fixed as 17 during this experiment, resulting in an overestimated channel estimate in the second part. 


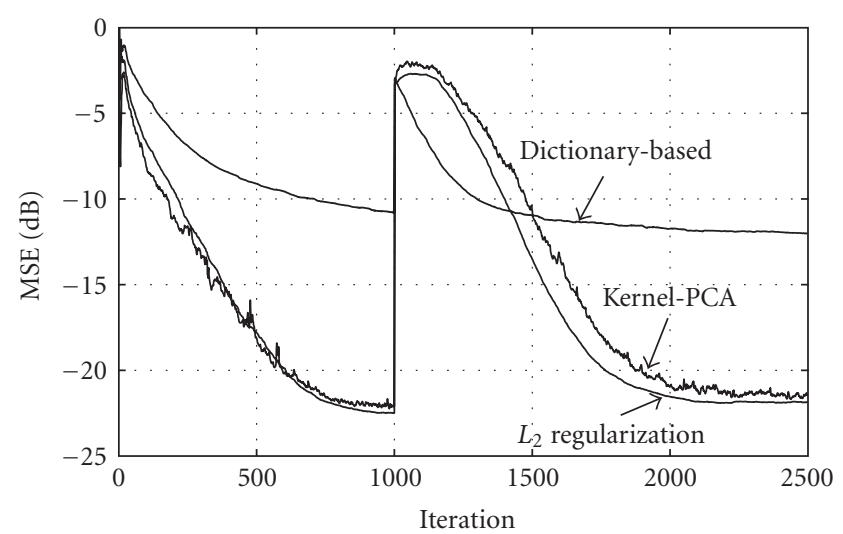

Figure 11: Wiener system MSE $e_{r}^{2}[n]$ obtained by adaptive identification of a Wiener system that exhibits an abrupt change and contains additive noise.

The identification results can be seen in Figure 11. As in the case of the static Wiener system, the dictionary-based kernel CCA algorithm obtains the worst performance, for reasons discussed earlier. The algorithm based on standard regularization and the one based on kernel PCA obtain very similar performance.

\subsubsection{Static Hammerstein system identification}

In this setup, we considered a static Hammerstein system consisting of the nonlinearity $f(x)=\tanh (x)$ followed by the linear channel $H(z)=1-0.4326^{-1}+0.3656 z^{-2}-0.3153 z^{-3}$. To the output of this system, $20 \mathrm{~dB}$ zero-mean additive white Gaussian noise was added. When applying the proposed kernel CCA-based algorithms to identify this system, the direct nonlinearity is estimated and an FIR estimate is made of the inverse linear channel which corresponds to an IIR filter. To adequately estimate this channel, the length of the direct FIR filter estimate was considerably increased.

The adaptive kernel CCA algorithms were applied with the following parameters:

(i) kernel CCA with standard regularization, $c=10^{-2}$, and a sliding window of 150 samples, using the Gaussian kernel function with kernel width $\sigma=0.2$;

(ii) kernel CCA based on kernel PCA using 10 eigenvectors, a 150-sample sliding window and the Gaussian kernel function with kernel width $\sigma=0.2$;

(iii) kernel CCA with dictionary-based sparsification, using accuracy parameter $v=10^{-2}$ and the same Gaussian kernel function.

In all three algorithms, the inverse linear channel was approximated as an FIR channel of length 15.

The MSE results for the Hammerstein system identification can be found in Figure 12. The observed MSE performances are similar to the observations already made for the previous examples. However, due to the different setup and the presence of noise, the obtained results are not as good as those of the identification of a static noiseless Wiener system (see Figure 8). Nevertheless, with the chosen parameters, the

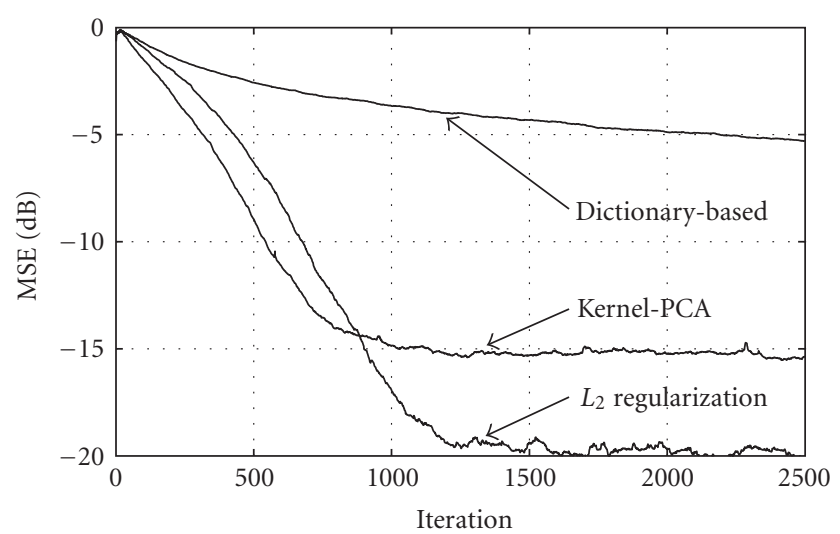

Figure 12: MSE $e_{r}^{2}[n]$ on the Hammerstein system's internal signal $r[n]$ for the three adaptive kernel CCA-based algorithms.

$L_{2}$ regularization-based kernel CCA algorithm is capable of attaining the $20 \mathrm{~dB}$ noise floor.

In all previous examples, the length $N_{w}$ of the sliding windows for the $L_{2}$ regularization-based kernel CCA and the kernel PCA-based kernel CCA was fixed as 150. Taking into account the number of eigenvectors used by the latter, both obtain a very similar computational complexity. The dictionary-based algorithm, on the other hand, is computationally much more attractive with its $O\left(M^{2}\right)$ complexity, but it is not capable of obtaining the same performance levels.

\section{CONCLUSIONS AND DISCUSSION}

In this paper, we have proposed a kernel-CCA-based framework to simultaneously identify the two parts of a Wiener or a Hammerstein system. Applying the correct restrictions on the solutions, it was shown how the proposed kernel CCA algorithm emerges as the logical solution to identify these nonlinear systems. Three different approaches to regularize the solutions of this kernel algorithm were discussed, resulting in three different implementations. In the second part of this paper, we showed how adaptive versions of these three algorithms could be derived, based on existing kernel RLS implementations and the reformulation of kernel CCA as a set of LS regression problems.

The proposed algorithms were compared in a series of batch and online experiments. The kernel CCA algorithm using the dictionary-based kernel RLS from [28] was found not suitable for adaptive kernel CCA since it is incapable of efficiently performing tracking. The kernel CCA algorithm using $L_{2}$ regularization and a sliding window obtained similar performance and computational cost as the kernel-PCAbased algorithm. These two algorithms showed to be successful in identifying both static and time-varying Wiener and Hammerstein systems.

Many directions for future research are open. The proposed methods can be used directly in problems with complex signals, such as communication signals, for instance, in the identification of nonlinear power amplifiers for OFDM systems [37]. Another possibility to explore is the application 
of kernel CCA to more complex cascade models such as the three-block Wiener-Hammerstein systems. And lastly, the problem of extending the proposed algorithms to blind identification can be considered.

\section{ACKNOWLEDGMENTS}

This work was supported by MEC (Ministerio de Educación y Ciencia, Spain) under Grants no. TEC2004-06451-C05-02, TEC2007-68020-C04-02 TCM, and FPU Grant no. AP20055366.

\section{REFERENCES}

[1] G. Giannakis and E. Serpedin, "A bibliography on nonlinear system identification," Signal Processing, vol. 81, no. 3, pp. 533-580, 2001.

[2] O. Nelles, Nonlinear System Identification, Springer, Berlin, Germany, 2000.

[3] G. Kechriotis, E. Zervas, and E. S. Manolakos, "Using recurrent neural networks for adaptive communication channel equalization," IEEE Transactions on Neural Networks, vol. 5, no. 2, pp. 267-278, 1994.

[4] N. P. Sands and J. M. Cioffi, "Nonlinear channel models for digital magnetic recording," IEEE Transactions on Magnetics, vol. 29, no. 6, pp. 3996-3998, 1993.

[5] A. Balestrino, A. Landi, M. Ould-Zmirli, and L. Sani, "Automatic nonlinear auto-tuning method for Hammerstein modeling of electrical drives," IEEE Transactions on Industrial Electronics, vol. 48, no. 3, pp. 645-655, 2001.

[6] D. Erdogmus, D. Rende, J. C. Principe, and T. F. Wong, "Nonlinear channel equalization using multilayer perceptrons with information-theoretic criterion," in Proceedings of the IEEE Workshop on Neural Networks for Signal Processing XI (NNSP '01), pp. 443-451, North Falmouth, Mass, USA, September 2001.

[7] T. Adali and X. Liu, "Canonical piecewise linear network for nonlinear filtering and its application to blind equalization," Signal Processing, vol. 61, no. 2, pp. 145-155, 1997.

[8] M. Pawlak, Z. Hasiewicz, and P. Wachel, "On nonparametric identification of Wiener systems," IEEE Transactions on Signal Processing, vol. 55, no. 2, pp. 482-492, 2007.

[9] J. Wang, A. Sano, T. Chen, and B. Huang, "Blind Hammerstein identification for MR damper modeling," in Proceedings of the American Control Conference (ACC '07), pp. 2277-2282, New York, NY, USA, July 2007.

[10] E. Aschbacher and M. Rupp, "Robustness analysis of a gradient identification method for a nonlinear wiener system," in Proceedings of the 13th IEEE Workshop on Statistical Signal Processing (SSP '05), vol. 2005, pp. 103-108, Bordeaux, France, July 2005.

[11] D. T. Westwick and R. E. Kearney, "Identification of a Hammerstein model of the stretch reflex EMG using separable least squares," in Proceedings of the 22nd Annual International Conference of the IEEE Engineering in Medicine and Biology Society (IEMBS '00), vol. 3, pp. 1901-1904, Chicago, Ill, USA, July 2000.

[12] J. E. Cousseau, J. L. Figueroa, S. Werner, and T. I. Laakso, "Efficient nonlinear Wiener model identification using a complexvalued simplicial canonical piecewise linear filter," IEEE Transactions on Signal Processing, vol. 55, no. 5, pp. 1780-1792, 2007.
[13] A. Taleb, J. Sole, and C. Jutten, "Quasi-nonparametric blind inversion of Wiener systems," IEEE Transactions on Signal Processing, vol. 49, no. 5, pp. 917-924, 2001.

[14] J. C. Gomez and E. Baeyens, "Subspace-based blind identification of IIR Wiener systems," in Proceedings of 15th European Signal Processing Conference (EUSIPCO '07), Poznań, Poland, September 2007.

[15] B. Schölkopf and A. J. Smola, Learning with Kernels, MIT Press, Cambridge, Mass, USA, 2002.

[16] S. Haykin, Neural Networks: A Comprehensive Foundation, Prentice-Hall, Englewood Cliffs, NJ, USA, 2nd edition, 1999.

[17] G. S. Kimeldorf and G. Wahba, "Some results on Tchebycheffian spline functions," Journal of Mathematical Analysis and Applications, vol. 33, no. 1, pp. 82-95, 1971.

[18] F. R. Bach and M. I. Jordan, "Kernel independent component analysis," Journal of Machine Learning Research, vol. 3, pp. 148, 2003.

[19] D. R. Hardoon, S. Szedmak, and J. Shawe-Taylor, "Canonical correlation analysis: an overview with application to learning methods," Tech. Rep. CSD-TR-03-02, Royal Holloway, University of London, Egham, Surrey, UK, 2003.

[20] P. L. Lai and C. Fyfe, "Kernel and nonlinear canonical correlation analysis," International Journal of Neural Systems, vol. 10, no. 5, pp. 365-377, 2000.

[21] H. Hotelling, "Relations between two sets of variates," Biometrika, vol. 28, pp. 321-377, 1936.

[22] S. Van Vaerenbergh, J. Vía, and I. Santamaría, “Online kernel canonical correlation analysis for supervised equalization of Wiener systems," in Proceedings of the International Joint Conference on Neural Networks (IJCNN '06), pp. 1198-1204, Vancouver, BC, Canada, July 2006.

[23] J. Vía, I. Santamaría, and J. Pérez, "Canonical correlation analysis (CCA) algorithms for multiple data sets: application to blind SIMO equalization," in Proceedings of the 13th European Signal Processing Conference (EUSIPCO '05), Antalya, Turky, September 2005.

[24] A. Tikhonov, "Solution of incorrectly formulated problems and the regularization method," Soviet Mathematics Doklady, vol. 4, pp. 1035-1038, 1963.

[25] S. Van Vaerenbergh, J. Vía, and I. Santamaría, "A slidingwindow kernel RLS algorithm and its application to nonlinear channel identification," in Proceedings of the IEEE International Conference on Acoustics, Speech and Signal Processing (ICASSP '06), vol. 5, pp. V789-V792, Toulouse, France, May 2006.

[26] K. I. Diamantaras and S. Y. Kung, Principal Component Neural Networks: Theory and Applications, John Wiley \& Sons, New York, NY, USA, 1996.

[27] B. Schölkopf, A. Smola, and K.-R. Müller, "Nonlinear component analysis as a kernel eigenvalue problem," Neural Computation, vol. 10, no. 5, pp. 1299-1319, 1998.

[28] Y. Engel, S. Mannor, and R. Meir, "The kernel recursive least squares algorithm," IEEE Transactions on Signal Processing, vol. 52, no. 8, pp. 2275-2285, 2004.

[29] S. Haykin, Adaptive Filter Theory, Prentice-Hall, Englewood Cliffs, NJ, USA, 4th edition, 2001.

[30] J. Vía, I. Santamaría, and J. Pérez, "A learning algorithm for adaptive canonical correlation analysis of several data sets," Neural Networks, vol. 20, no. 1, pp. 139-152, 2007.

[31] A. Pezeshki, L. L. Scharf, M. R. Azimi-Sadjadi, and Y. Hua, "Two-channel constrained least squares problems: solutions using power methods and connections with canonical coordinates," IEEE Transactions on Signal Processing, vol. 53, no. 1, pp. 121-135, 2005. 
[32] J. Vía, I. Santamaría, and J. Pérez, "A robust RLS algorithm for adaptive canonical correlation analysis," in Proceedings of the IEEE International Conference on Acoustics, Speech and Signal Processing (ICASSP '05), vol. 4, pp. 365-368, Philadelphia, Pa, USA, March 2005.

[33] J. Kivinen, A. J. Smola, and R. C. Williamson, "Online learning with kernels," IEEE Transactions on Signal Processing, vol. 52, no. 8, pp. 2165-2176, 2004.

[34] S. Van Vaerenbergh, J. Vía, and I. Santamaría, "Nonlinear system identification using a new sliding-window kernel RLS algorithm," Journal of Communications, vol. 2, no. 3, pp. 1-8, 2007.

[35] L. Hoegaerts, L. De Lathauwer, I. Goethals, J. A. K. Suykens, J. Vandewalle, and B. De Moor, "Efficiently updating and tracking the dominant kernel principal components," Neural Networks, vol. 20, no. 2, pp. 220-229, 2007.

[36] E. Andelić, M. Schafföner, M. Katz, S. E. Krüger, and A. Wendemuth, "Kernel least-squares models using updates of the pseudoinverse," Neural Computation, vol. 18, no. 12, pp. 2928 2935, 2006.

[37] I. Santamaría, J. Ibáñez, M. Lázaro, C. Pantaleón, and L. Vielva, "Modeling nonlinear power amplifiers in OFDM systems form subsampled data: a comparative study using real measurements," EURASIP Journal on Applied Signal Processing, vol. 2003, no. 12, pp. 1219-1228, 2003. 